\title{
ON THE HARDY-LITTLEWOOD MAXIMAL FUNCTION AND SOME APPLICATIONS \\ BY
}

\author{
C. J. NEUGEBAUER
}

\begin{abstract}
With a monotone family $F=\left\{S_{\alpha}\right\}, S_{\alpha} \subset \mathbf{R}^{n}$, we associate the HardyLittlewood maximal function $M_{F} f(x)=\sup _{\alpha}\left(1 /\left|S_{\alpha}\right|\right) \int_{S_{\alpha}+x}|f|$. In general, $M_{F}$ is not weak type (1.1). However, if we replace in the denominator $S_{\alpha}$ by $S_{\alpha}^{*}=\{x-$ $\left.y: x, y \in S_{\alpha}\right\}$, and denote the resulting maximal function by $M_{F}^{*}$, then $M_{F}^{*}$ is weak type $(1,1)$ with weak type constant 1 .
\end{abstract}

1. Let $F=\left\{S_{\alpha}\right\}, \alpha \in \Gamma$, be a family of measurable sets in $\mathbf{R}^{n}, 0<\left|S_{\alpha}\right|<\infty$, and for $f: \mathbf{R}^{n} \rightarrow \mathbf{R}$ measurable, let $M_{F} f(x)=\sup _{\alpha}\left(1 /\left|S_{\alpha}\right|\right) \int_{S_{\alpha}+x}|f(t)| d t$, the HardyLittlewood maximal function relative to $F$. For many important families $F, M_{F}$ is weak type $(1,1)$ and consequently strong type $(p, p), 1<p<\infty$. This is true if $S_{\alpha}=\{|x|<\alpha\}$, or if $S_{\alpha}$ is the cube of side length $\alpha$ centered at the origin, or if $\left\{S_{\alpha}\right\}$ is a nested family of rectangles with sides parallel to the coordinate axes (see [7]). However, one cannot go much beyond families of this kind and still expect a weak type result. If, for example, $F$ is the collection of all oriented rectangles, $M_{F}$ is not weak type $(1,1)$ (see [6]), even though it is strong type $(p, p), 1<p<\infty$, and if $F$ is the collection of all rectangles, not even this holds (see [4]).

It is well known that the maximal function is an important tool (i) in obtaining pointwise bounds for convolutions [7], (ii) in problems of convergence a.e. and (iii) in the theory of differentiability [4]. It is thus of interest to study $M_{F}$ for relatively arbitrary $F$.

For the study of $M_{F}$, as we have seen, one needs to restrict $F=\left\{S_{\alpha}\right\}$, and a natural minimal condition is that $\alpha<\beta$ implies $S_{\alpha} \subset S_{\beta}$. We will give, however, an example (due to $R$. Hunt) which shows that $M_{F}$ need not be weak type. This forces us to introduce a modified maximal function $M_{F}^{*}$ which is equivalent with $M_{F}$ whenever $F$ is one of the classical families, and which is weak type $(1,1)$. This is done in the next section, and the remaining sections deal with some applications and $\mathbf{R}$. Hunt's example.

2. For $E \subset \mathbf{R}^{n}$, let $E^{*}=E-E=\{x-y: x, y \in E\}$. We need the following lemma.

LemMa. Let $E \subset \mathbf{R}^{n}$ be measurable with $0<|E|<\infty$. If every point of $E$ is a point of density of $E$, then $E^{*}$ is open.

Received by the editors November 6, 1978.

AMS (MOS) subject classifications (1970). Primary 42A96; Secondary 28A15.

Key words and phrases. Hardy-Littlewood maximal function, monotone family, weak type.

(c) 1980 American Mathematical Society 0002-9947/80/0000-0205/\$02.75 
Proof. We give the short proof (which is standard) for the sake of completeness. We denote by $d(x, E)=\lim |E \cap Q||Q|^{-1}$, as $|Q| \rightarrow 0$, where $Q$ is a cube, $x \in Q$. The hypothesis says that $d(x, E)=1, x \in E$. We consider the continuous function $\psi(z)=\int_{\mathbf{R}^{n}} \chi_{E}(z+t) \chi_{E}(t) d t=\chi_{E} * \chi_{E}(z)$, and we need to show that for $z_{0} \in E^{*}$, $\psi\left(z_{0}\right)>0$. If $A=\left\{\tau: x_{0}+\tau \in E\right\}, B=\left\{\tau: y_{0}+\tau \in E\right\}$, where $z_{0}=x_{0}-y_{0}$, then $d(0, A)=d(0, B)=1$, and hence $d(0, A \cap B)=1$. For $\tau \in A \cap B$, and $t=$ $y_{0}+\tau$ we get $z_{0}+t=x_{0}+\tau \in E$ and hence $\psi\left(z_{0}\right)>\int_{A \cap B+y_{0}} \chi_{E}\left(z_{0}+t\right) \chi_{E}(t) d t$ $>0$.

For $F=\left\{T_{\gamma}\right\}, \gamma \in \Gamma$, a family of measurable sets in $\mathbf{R}^{n}$ with (i) $0<\left|T_{\gamma}\right|<\infty$, let

$$
M_{F}^{*} f(x)=\sup _{\gamma} \frac{1}{\left|T_{\gamma}^{*}\right|} \int_{T_{\gamma}+x}|f(t)| d t .
$$

By the above lemma we may assume that $T_{\gamma}^{*}$ is open, and we shall do so throughout the paper. In addition to (i) above, we need to assume that there is a map $\sigma: \Gamma \rightarrow(0, \infty]$ such that (ii) $\sigma\left(\gamma_{1}\right)<\sigma\left(\gamma_{2}\right)$ implies $T_{\gamma_{1}} \subset T_{\gamma_{2}}$, (iii) $\sigma\left(\gamma_{i}\right) \uparrow \sigma$ implies there exists $\gamma \in \Gamma$ such that $\sigma=\sigma(\gamma)$ and $T_{\gamma_{i}} \uparrow T_{\gamma}$.

REMARK. If $T_{\gamma}$ is convex, then $\left|T_{\gamma}^{*}\right|=2^{n}\left|T_{\gamma}\right|$, and hence $M_{F}^{*}$ is equivalent with $\boldsymbol{M}_{\boldsymbol{F}}$.

THEOREM 1. Under the above conditions on $F$,

$$
\left|\left\{x: M_{F}^{*} f(x)>y\right\}\right|<\|f\|_{1} / y, \quad y>0 \text {, i.e., } M_{F}^{*} \text { is weak type }(1,1) .
$$

REMARK. The reader will notice that the proof of Theorem 1 is a refinement of the covering and weak type theorems in $\$ 2$ of [5].

Proof. We will first assume that $T_{\gamma} \subset\{|x|<N\}, \gamma \in \Gamma$. We fix $y>0$ and let $E=\left\{x: M_{F}^{*} f(x)>y\right\}$. For $r>0$, let $E_{r}=E \cap\{|x|<r\}$. We will show that $\left|E_{r}\right|<\|f\|_{1} / y$, and this will prove the theorem under the hypothesis of boundedness of $T_{\gamma}$.

We may clearly assume that the map $\sigma$ is bounded on $\Gamma$; otherwise, the map $\sigma^{\prime}(\gamma)=\tan ^{-1} \sigma(\gamma)$ produces a reindexing with the required property.

We note that $E_{r}$ is contained in the set $A_{0}$ of all $x$ for which $|x|<r$ and, for some $\gamma \in \Gamma, \int_{T_{\gamma}+x}|f(t)| d t>y\left|T_{\gamma}^{*}\right|$. For $x \in A_{0}$, let $\tau(x)=\sup \left\{\sigma(\gamma): \int_{T_{\gamma}+x}|f(t)| d t\right.$ $\left.>y\left|T_{\gamma}^{*}\right|\right\}$. The number $\tau(x)<\infty$, and there is a sequence $\left\{\gamma_{j}\right\}$ such that $\sigma\left(\gamma_{j}\right) \uparrow \tau(x)$, and $\int_{T_{\gamma_{j}+x}}|f(t)| d t \geqslant y\left|T_{\gamma_{j}}^{*}\right|$. By hypothesis, there is $\gamma \in \Gamma$ such that $\tau(x)=\sigma(\gamma)$ and $T_{\gamma,} \uparrow T_{\gamma}$, from which $\int_{T_{\gamma}+x}|f(t)| d t>y\left|T_{\gamma}^{*}\right|$.

We let now $\tau_{1}=\sup \left\{\tau(x): x \in A_{0}\right\}$. Then $\tau_{1}<\infty$ and we have a sequence $\left\{x_{j}\right\} \subset A_{0}$ such that $\tau\left(x_{j}\right) \uparrow \tau_{1}$ and $x_{j} \rightarrow x_{1}$. As above, $\tau\left(x_{j}\right)=\sigma\left(\gamma_{j}\right)$, and there is $\gamma_{1} \in \Gamma$ such that $\tau_{1}=\sigma\left(\gamma_{1}\right), T_{\gamma_{j}} \uparrow T_{\gamma_{1}}$, and $\int_{T_{\gamma_{1}}+x_{1}}|f(t)| d t>y\left|T_{\gamma_{1}}^{*}\right|$. Thus $x_{1} \in A_{0}$. Let now $A_{1}=A_{0} \backslash\left(T_{\gamma_{1}}^{*}+x_{1}\right)$, and let $\tau_{2}=\sup \left\{\tau(x): x \in A_{1}\right\}$. Then $\tau_{1}>\tau_{2}$, and as before there is $\left\{x_{j}\right\} \subset A_{1}$ such that $x_{j} \rightarrow x_{2}, \tau\left(x_{j}\right) \uparrow \tau_{2}$. Arguing as above there is $\gamma_{2} \in \Gamma$ such that $\tau_{2}=\sigma\left(\gamma_{2}\right)$ and $\int_{T_{\gamma_{2}}+x_{2}}|f(t)| d t>y\left|T_{\gamma_{2}}^{*}\right|$. Hence $x_{2} \in A_{0}$, and since $A_{1}$ is closed in $A_{0}$ (recall $T_{\gamma_{1}}^{*}$ is open), $x_{2} \in A_{1}$. We also note that $T_{\gamma_{2}} \subset T_{\gamma_{1}}$. 
We continue this process and obtain sequences $\left\{x_{j}\right\},\left\{T_{\gamma_{j}}\right\},\left\{A_{j}\right\}$ such that

(1) $j<k$ implies $T_{\gamma_{j}} \supset T_{\gamma_{k}}$,

(2) $x_{j+1} \in A_{j}$ and $A_{j}=A_{j-1} \backslash\left(T_{\gamma_{j}}^{*}+x_{j}\right)$,

(3) $\int_{T_{y_{j}}+x_{j}}|f(t)| d t>y\left|T_{\gamma_{j}}^{*}\right|$.

We claim now that for $j \neq k,\left(x_{j}+T_{\gamma_{j}}\right) \cap\left(x_{k}+T_{\gamma_{k}}\right)=\varnothing$. Assume that $j<k$, and $x_{j}+t_{j}=x_{k}+t_{k}, t_{j} \in T_{\gamma_{j}}, t_{k} \in T_{\gamma_{k}}$. Then $x_{k} \in x_{j}+\left(T_{\gamma_{j}}-T_{\gamma_{k}}\right) \subset x_{j}+T_{\gamma_{j}}^{*}$ by (1). But by (2), $x_{k} \in A_{k-1}$ and $A_{k-1} \cap\left(T_{\gamma_{j}}^{*}+x_{j}\right)=\varnothing$.

We assert next that $\cap A_{j}=\varnothing$. Assume that $z \in \cap A_{j}$. Then $\tau(z)>0$ (see the beginning of the proof for the definition of $\tau(z))$, and as observed there $\tau(z)=\sigma(\gamma)$ and $\int_{T_{\gamma}+z}|f(z)| d t>y\left|T_{\gamma}^{*}\right|$. Since $\tau_{j}>\tau(z)$, we have $T_{\gamma_{j}} \supset T_{\gamma}$, and thus, if $F=$ $\cup\left(x_{j}+T_{\gamma_{j}}\right),|F|=\infty$. Also $T_{\gamma_{j}} \subset T_{\gamma_{1}}$ so that $\cup T_{\gamma_{j}} \subset T_{\gamma_{1}}$. Since $\left|x_{j}\right|<r$ we see that $F \subset T_{\gamma_{1}}+\{|x|<r\} \subset\{|x|<N\}+\{|x|<r\}$, contradicting $|F|=\infty$.

Finally, since $E_{r} \subset \cup\left(x_{j}+T_{\gamma_{j}}^{*}\right)$, we obtain

$$
\left|E_{r}\right| \leqslant \Sigma\left|T_{\gamma_{j}}^{*}\right| \leqslant \frac{1}{y} \Sigma \int_{T_{\gamma_{j}+x_{j}}}|f(t)| d t \leqslant \frac{\|f\|_{1}}{y} .
$$

We remove now the restriction that each $T_{\gamma} \subset\{|x|<N\}$. We let $F_{N}=\left\{T_{\gamma}^{N}\right\}$, where $T_{\gamma}^{N}=T_{\gamma} \cap\{|x|<N\}$. Then

$$
M_{N} f(x) \equiv \sup _{\gamma} \frac{1}{\left|T_{\gamma}^{*}\right|} \int_{T_{\gamma}^{N}+x}|f(t)| d t \leqslant \sup _{\gamma} \frac{1}{\left|T_{\gamma}^{N *}\right|} \int_{T_{\gamma}^{N}+x}|f(t)| d t,
$$

and since the first part of the proof applies to $F_{N},\left|\left\{x: M_{N} f(x)>y\right\}\right|<\|f\|_{1} / y$. Since $M_{N} f(x) \uparrow M_{F}^{*} f(x)$, we see that $\left|\left\{x: M_{F}^{*} f(x)>y\right\}\right|=\lim _{N \rightarrow \infty}\left|\left\{x: M_{N} f(x)>y\right\}\right|$ $<\|f\|_{1} / y$. This completes the proof of Theorem 1 .

Corollary 1. $\left\|M_{F}^{*} f\right\|_{p}<A_{p}\|f\|_{p}, 1<p<\infty$, where $A_{p}$ depends only on $p$. In particular, $A_{p}$ does not depend on $F, f$.

Corollary 2. $\left|\left\{x: M_{F}^{*} f(x)>y\right\}\right|<(2 / y) \int_{|f(t)|>y / 2}|f(t)| d t$.

Proof. Let $f=f_{1}+f_{2}$, where $f_{1}(x)=f(x)$, whenever $|f(x)|>y / 2$, and $f_{1}(x)=0$ elsewhere. Then $|f(x)|<\left|f_{1}(x)\right|+y / 2$. Hence $\left\{x: M_{F}^{*} f(x)>y\right\} \subset\left\{x: M_{F}^{*} f_{1}(x)>\right.$ $y / 2\}$, and thus

$$
\left|\left\{x: M_{F}^{*} f(x)>y\right\}\right|<\frac{2}{y}\left\|f_{1}\right\|_{1}=\frac{2}{y} \int_{|f(t)|>y / 2}|f(t)| d t .
$$

We give now an important special example of Theorem 1. We let $F=\left\{S_{\alpha}\right\}$, $\alpha \in \Gamma, \Gamma \subset(0, \infty)$, such that (i) $0<\left|S_{\alpha}\right|<\infty$, (ii) $\alpha<\beta$ implies $S_{\alpha} \subset S_{\beta}$.

THEOREM 2. $\left|\left\{x: M_{F}^{*} f(x)>y\right\}\right|<\|f\|_{1} / y$.

Proof. Let $\Gamma^{\prime}=\left\{\tau: \exists\left\{\alpha_{j}\right\} \subset \Gamma\right.$ such that $\left.\alpha_{j} \uparrow \tau\right\}$. If $\tau \in \Gamma^{\prime} \backslash \Gamma$, let $S_{\tau}=\cup_{\alpha<\tau} S_{\alpha}$, $\alpha \in \Gamma$. We let $\tilde{\Gamma}=\Gamma^{\prime} \times[0,1]$, and for $\gamma=(\alpha, \beta)$ in $\tilde{\Gamma}$ we define $T_{\gamma}$ as follows. If $\beta=0, T_{\gamma}=\cup_{\tau<\alpha} S_{\tau}$, and if $0<\beta<1, T_{\gamma}=S_{\alpha}$. We set $\tilde{F}=\left\{T_{\gamma}\right\}$, and we define $\sigma: \tilde{\Gamma} \rightarrow(0, \infty)$ by $\sigma(\alpha, \beta)=\alpha$. It is easily checked that Theorem 1 applies to $\tilde{F}$ and hence

$$
\left|\left\{x: M_{F}^{*} f(x)>y\right\}\right|<\left|\left\{x: M_{F}^{*} f(x)>y\right\}\right|<\|f\|_{1} / y \text {. }
$$


3. The hypothesis in Theorem 2 that the family $F=\left\{S_{\alpha}\right\}$ be monotone can be somewhat weakened. For $E \subset \mathbf{R}^{n}$ and $k=\left(k_{1}, \ldots, k_{n}\right), k_{i}>0$, let $k E=$ $\left\{\left(k_{1} x_{1}, \ldots, k_{n} x_{n}\right):\left(x_{1}, \ldots, x_{n}\right) \in E\right\}$.

THEOREM 3. Let $F=\left\{S_{\alpha}\right\}, \alpha \in \Gamma$, have the property that there is $k$ such that $\alpha^{\prime}, \alpha^{\prime \prime}<\beta$ in $\Gamma$ implies $S_{\alpha^{\prime}}-S_{\alpha^{\prime \prime}} \subset k S_{\beta}^{*}$. Then

$$
\left|\left\{x: M_{F}^{*} f(x)>y\right\}\right|<\frac{k_{1} \cdots k_{n}}{y}\|f\|_{1} \text {. }
$$

Proof. Let $T_{\beta}=\cup_{\alpha<\beta} S_{\alpha}$. Since we may assume that every point of $S_{\alpha}$ is a point of density of $S_{\alpha}$, the set $T_{\beta}$ is measurable. Let $F^{\prime}=\left\{T_{\beta}\right\}$. It is clear that $F^{\prime}$ is monotone, and $T_{\beta}^{*} \subset \cup_{\alpha^{\prime}, \alpha^{\prime \prime}<\beta}\left(S_{\alpha^{\prime}}-S_{\alpha^{\prime \prime}}\right) \subset k S_{\beta}^{*}$. From this we get $M_{F}^{*} f(x)<$ $k_{1} \cdots k_{n} M_{F}^{*} f(x)$, and Theorem 2 completes the proof.

COROLlaRY. If $F=\left\{S_{\alpha}\right\}$ has the property that there is $k$ so that $\alpha<\beta$ implies $k S_{\beta} \supset S_{\alpha}$, then the above weak type inequality holds.

Proof. Simply observe that $\alpha^{\prime}, \alpha^{\prime \prime}<\beta$ implies $S_{\alpha^{\prime}}-S_{\alpha^{\prime \prime}} \subset k S_{\beta}^{*}$.

REMARK. The special case of the above corollary with $F$ a family of oriented rectangles centered at the origin can be found in [1] with a different weak type constant.

4. The following example, due to $R$. Hunt, shows that for a nested family $F=\left\{S_{\alpha}\right\}$ (as in Theorem 2) the maximal function $M_{F} f(x)=$ $\sup _{\alpha}\left(1 /\left|S_{\alpha}\right|\right) \int_{S_{\alpha}+x}|f(t)| d t$ need not be weak type $(1,1)$.

Let $S_{N}=\cup_{j=N}^{\infty}\left(2^{-j}, 2^{-j}+2^{-2 j}\right)$ in $\mathbf{R}$, so that $S_{N+1} \subset S_{N}$. Let $F=\left\{S_{N}\right\}$, and note that $\left|S_{N}\right|<2^{-2 N+1}$. Let $f_{N}$ be the characteristic function of $\left(0,2^{-2 N-1}\right)$. Then $\|f\|_{1}=2^{-2 N-1}$. It is readily checked that

$$
\begin{aligned}
x \in\left(-2^{-N-1},-2^{-N-1}+2^{-2 N-2}\right) & \Rightarrow M_{F} f_{N}(x)>2^{-2 N-2} /\left|S_{N+1}\right|>1 / 2, \\
x \in\left(-2^{-N-2},-2^{-N-2}+2^{-2 N-2}\right) & \Rightarrow M_{F} f_{N}(x)>2^{-2(N+2)} /\left|S_{N+2}\right|>1 / 2, \\
& \vdots \\
x \in\left(-2^{-(N+N+1)}, 0\right) & \Rightarrow M_{F} f_{N}(x)>1 / 2 .
\end{aligned}
$$

Hence $\left|\left\{x: M_{F} f_{N}(x)>1 / 2\right\}\right|>N \cdot 2^{-2 N-2}=N\left\|f_{N}\right\|_{1} / 2$, and $M_{F}$ is not weak type $(1,1)$. Incidentally, $M_{F}$ is not weak type $(p, p), 1<p<\infty$.

The above example raises the question for which class of functions is $M_{F} f$ weak type $(1,1)$. For $F=\left\{S_{\alpha}\right\}, \alpha \in \Gamma$, a monotone family, and $\mu>0$ a measure on $\mathbf{R}^{n}$, let us define $\mu_{F}(t)=\sup \mu\left(S_{\alpha}^{*}+x\right) /\left|S_{\alpha}\right|$, where the sup is taken over all $\alpha, x$ for which $t \in S_{\alpha}+x$. Since $S_{\alpha}^{*}$ is open (recall that we always assume that $x \in S_{\alpha}$ is a point of density of $S_{\alpha}$ ), it follows easily that $\mu_{F}$ is lower semicontinuous and hence measurable.

THEOREM 4. Under the above conditions

$$
\mu\left\{x: M_{F} f(x)>y\right\}<\frac{1}{y} \int_{\mathbf{R}^{n}}|f(t)| \mu_{F}(t) d t .
$$


Proof. As in Theorem 2 we extend $F$ to a family $\tilde{F}=\left\{T_{\gamma}\right\}, \gamma \in \tilde{\Gamma}$, for which there is a mapping $\sigma: \tilde{\Gamma} \rightarrow(0, \infty)$ so that the hypotheses of Theorem 1 hold. Then as in the proof of Theorem 1 we select $\left\{T_{\gamma_{j}}\right\},\left\{x_{j}\right\}$ and obtain

$$
\begin{aligned}
\frac{1}{y} \int_{\mathbf{R}^{n}}|f(t)| \mu_{F}(t) d t & \geqslant \frac{1}{y} \sum \int_{T_{\gamma_{j}+x_{j}}}|f(t)| \mu_{F}(t) d t \\
& \geqslant \frac{1}{y} \Sigma \frac{\mu\left(T_{\gamma_{j}}^{*}+x_{j}\right)}{\left|T_{\gamma_{j}}\right|} \int_{T_{\gamma^{+x_{j}}}}|f(t)| d t>\Sigma \mu\left(T_{\gamma_{j}}^{*}+x_{j}\right) \\
& \geqslant \mu\left\{x: M_{F} f(x)>y\right\} .
\end{aligned}
$$

In the second inequality we used the fact that $\mu_{F}(t)=\mu_{\tilde{F}}(t)$ which follows easily from the definition of $\tilde{F}$ in the proof of Theorem 2 .

5. In this section we will present some applications of Theorem 2 concerning pointwise boundedness of convolutions and approximate identities.

The problem about convolutions which we will discuss can be put in the following way. Suppose $\lim \phi_{n} * g(x)$ exists for every $x$ and $g \in C_{c}\left(\mathbf{R}^{n}\right)$. Under what conditions does it follow that $\lim \phi_{n} * f(x)$ exists for a.e. $x$ and $f \in L^{p}\left(\mathbf{R}^{n}\right)$ (see e.g. [3]). The hypothesis on $C_{c}\left(\mathbf{R}^{n}\right)$ implies that $\left\|\phi_{n}\right\|_{1}<K<\infty$ (by the Banach-Steinhaus theorem). Our condition will be a more stringent requirement on the norm.

Let $F=\left\{S_{\alpha}\right\}, \alpha \in \Gamma \subset(0, \infty)$, be a family of measurable sets satisfying (i) $0<\left|S_{\alpha}\right|<\infty$, (ii) $\alpha<\beta$ implies $S_{\alpha} \subset S_{\beta}$. Let $\sigma(F)$ be the collection of all simple functions $s>0$ which can be written as $s=\Sigma c_{j} \chi_{A_{j}}, c_{j}>0$, and $A_{j} \subset-S_{\alpha_{j}}$ for some $\alpha_{j} \in \Gamma$, where $-E=\{x:-x \in E\}$. We set $\|s\|=\inf \Sigma c_{j}\left|S_{\alpha_{j}}^{*}\right|$, where the inf is extended over all representations $s=\Sigma c_{j} \chi_{1_{1}}$ and all sequences $\left\{S_{\alpha_{j}}\right\} \subset F$ with $A_{j} \subset-S_{\alpha,}$. We note that $\|s\|>\|s\|_{1}$. Denote now by $L(F)$ all functions $\phi>0$ for which there is a sequence $\left\{s_{n}\right\} \subset \sigma(F)$ such that $s_{n} \uparrow \phi$, and let $\|\phi\|_{F}=$ inf $\lim \inf \left\|s_{n}\right\|$, where the inf is extended over all such $\left\{s_{n}\right\}$. Again, $\|\phi\|_{F}>\|\phi\|_{1}$.

As an example, let $\phi>0, \phi \in L^{1}\left(\mathbf{R}^{n}\right)$, and let $S_{\alpha}=\left\{x: \phi(x)>\alpha^{-1}\right\}$. It is easily verified that $\phi \in L(F)$, where $F=\left\{S_{\alpha}\right\}$.

Lemma. Let $\phi \in L(F)$. Then $|\phi * f(x)|<\|\phi\|_{F} M_{F}^{*} f(x)$.

Proof. Let $s \in \sigma(F), s<\phi$. Choose a representation $s=\Sigma c_{j} \chi_{1_{1}}, c_{j}>0$, and $A_{j} \subset-S_{\alpha_{j}}$. Then $|s * f(x)|<\sum c_{j} \int|f(t)| \chi_{1_{j}}(x-t) d t<\sum c_{j} \int_{S_{\alpha_{j}}+x}|f(t)| d t<$ $\sum c_{j}\left|S_{\alpha,}^{*}\right| M_{F}^{*} f(x)$. Thus $|s * f(x)| \leqslant\|s\| M_{F}^{*} f(x)$, and by the monotone convergence theorem the lemma follows.

Let $\left\{\phi_{n}\right\}, \phi_{n}>0$, be a sequence of measurable functions. We say that a family $F=\left\{S_{\alpha}\right\}$, satisfying (i), (ii) above, dominates $\left\{\phi_{n}\right\}$ if $\left\{\phi_{n}\right\} \subset L(F)$ and $\left\|\phi_{n}\right\|_{F}<K$ $<\infty, n=1,2, \ldots$

TheOREM 5. If $F=\left\{S_{\alpha}\right\}$ dominates $\left\{\phi_{n}\right\}$ and if $\lim \phi_{n} * g(x)$ exists for a.e. $x$ and $g \in C_{c}\left(\mathbf{R}^{n}\right)$, then $\lim \phi_{n} * f(x)$ exists for a.e. $x$ if $f \in L^{p}\left(\mathbf{R}^{n}\right), 1<p<\infty$. 
Proof. From the lemma, $\sup _{n}\left|\phi_{n} * f(x)\right|<K M_{F}^{*} f(x)$. For $g \in C_{c}\left(\mathbf{R}^{n}\right)$ we have

$$
\begin{aligned}
\mid\left\{x: \lim \sup \phi_{n}\right. & \left.* f(x)-\lim \inf \phi_{n} * f(x)>\eta\right\} \mid \\
& =\left|\left\{x: \lim \sup \phi_{n} *(f-g)(x)-\lim \inf \phi_{n} *(f-g)(x)>\eta\right\}\right| \\
& <\left|\left\{x: M_{F}^{*}(f-g)(x)>\eta / 2 K\right\}\right| .
\end{aligned}
$$

By Theorem 2, $M_{F}^{*}$ is weak type $(p, p)$ and hence the measure of the above set $<A_{p}\|f-g\|_{p}^{p} / \eta^{p}$, which can be made as small as we wish.

Remark. (1) If $\lim \phi_{n} * g(x)=g(x)$, for a.e. $x$ and $g \in C_{c}$, then $\lim \phi_{n}^{*} f(x)=$ $f(x)$, a.e. $x$ and $f \in L^{p}, 1<p<\infty$.

(2) As an example illustrating Theorem 3 , let $\left\{\phi_{j}\right\}$ be a sequence of nonnegative functions such that $\phi_{j}(x)<\psi(x), x \in \mathbf{R}^{n}, j=1,2, \ldots, \psi(x) \in L^{1}\left(\mathbf{R}^{n}\right), \psi(x)=$ $\psi_{0}(|x|)$ and $\psi_{0}(r)$ is nonincreasing. Then the family $F$ of all spheres centered at 0 dominates $\left\{\phi_{n}\right\}$. This covers the classical case (see e.g. [7, p. 62]).

For the study of approximate identities, let $\phi>0$ on $\mathbf{R}^{n}, \int_{\mathbf{R}^{n}} \phi(t) d t=1$ and let $\phi_{e}(t)=\varepsilon^{-n} \phi(t / \varepsilon)$. Then $\int \phi_{\varepsilon} d t=1$.

Let $F=\left\{S_{\alpha}\right\}$ be a nested family as above and satisfying $\varepsilon F \subset F, 0<\varepsilon<1$, and $\phi \in L(F)$. Here $\varepsilon F \subset F$ means $\varepsilon S_{\alpha} \in F$ for $S_{\alpha} \in F$.

TheOREM 6. If $\|\phi\|_{F}<\infty$, then $\phi_{\varepsilon} * f(x) \rightarrow f(x)$ as $\varepsilon \rightarrow 0$ for a.e. $x, f \in L^{p}\left(\mathbf{R}^{n}\right)$, $1<p<\infty$.

Proof. It is easily verified that $\phi_{e} * g(x) \rightarrow g(x), x \in \mathbf{R}^{n}, g \in C_{c}\left(\mathbf{R}^{n}\right)$. By the lemma, $\left|\phi_{\varepsilon} * f(x)\right|<\left\|\phi_{e}\right\|_{F} M_{F}^{*}(x)$. One readily verifies that $\left\|\phi_{\varepsilon}\right\|_{F}=\|\phi\|_{F}$, and thus Theorem 3 completes the proof.

6. In this section we give an application to the differentiability of the integral. We let $0<|B|<\infty$ and $B_{\varepsilon}=\varepsilon \cdot B, 0<\varepsilon<1$. Assume that (i) $B \subset \cup B_{k}$, (ii) for each $k$, the family $F_{k}=\left\{\varepsilon B_{k}\right\}, 0<\varepsilon<1$, is nested, i.e., $\varepsilon_{1}<\varepsilon_{2}$ implies $\varepsilon_{1} B_{k}<$ $\varepsilon_{2} B_{k}$, (iii) $\Sigma\left|B_{k}^{*}\right|<\infty$. If for example $B$ is starlike and symmetric about 0 , then $B_{k}$ can be chosen to be a rectangle so that the above conditions hold (see [2]).

THEOREM 7. Under the above conditions, if $f^{*}(x)=\sup _{e}\left(1 /\left|B_{\varepsilon}\right|\right) \int_{B_{\varepsilon}+x}|f(t)| d t$, then $\left\|f^{*}\right\|_{p}<A_{p}\|f\|_{p}, 1<p<\infty$.

Proof. Let $\varepsilon B_{k}=B_{k e}$ and $M_{k}^{*} f(x)=\sup _{\varepsilon}\left(1 /\left|B_{k e}^{*}\right|\right) \int_{B_{k e}+x}|f(t)| d t$. Then

$$
\begin{aligned}
\frac{1}{\left|B_{e}\right|} \int_{B_{e}+x}|f(t)| d t & <\frac{1}{\left|B_{e}\right|} \Sigma \int_{B_{k e}+x}|f(t)| d t \\
& <\frac{1}{\left|B_{e}\right|} \sum\left|B_{k e}^{*}\right| M_{k}^{*} f(x)=\frac{1}{|B|} \Sigma\left|B_{k}^{*}\right| M_{k}^{*} f(x) .
\end{aligned}
$$

Hence $f^{*}(x)<(1 /|B|) \Sigma\left|B_{k}^{*}\right| M_{k}^{*} f(x)$, and Corollary 1 completes the proof.

REMARK. (1) The above result generalizes part of Theorem $A$ in [1].

(2) If the condition $\Sigma\left|B_{k}^{*}\right|<\infty$ is replaced by $\left|B_{k}^{*}\right| \rightarrow 0$ and $\Sigma\left|B_{k}^{*}\right||\log | B_{k}^{*} \|<$ $\infty$, then by $[4$, p. 145$], f^{*}$ is also weak type $(1,1)$. 


\section{REFERENCES}

1. L. A. Cafarelli and C. P. Calderón, Weak type estimates for the Hardy-Littlewood maximal function, Studia Math. 49 (1974), 217-223.

2. C. P. Calderón, Differentiation through star-like sets in $\mathbf{R}^{n}$, Studia Math. 48 (1973), 1-13.

3. R. E. Edwards and E. Hewitt, Pointwise limit for sequences of conoolution operators, Acta Math. 113 (1965), 181-218.

4. M. Guzmán, Differentiation of integrals in $\mathbf{R}^{n}$, Lecture Notes in Math., vol. 481, Springer-Verlag, Berlin and New York, 1975.

5. N. M. Rivière, Singular integrals and multiplier operators, Ark. Mat. 9 (1971), 243-278.

6. S. Saks, Remark on the differentiability of the Lebesgue indefinite integral, Fund. Math. 22 (1934), 257-261.

7. E. M. Stein, Singular integrals and differentiability properties of functions, Princeton Univ. Press, Princeton, N. J., 1970.

Division of Mathematical Sciences, PURdue University, West LapayetTe, Indina 47907 